\title{
Fibroblast growth factor 21 protects rat cardiomyocytes from endoplasmic reticulum stress by promoting the fibroblast growth factor receptor 1-extracellular signal-regulated kinase 1/2 signaling pathway
}

\author{
PINGPING LIANG ${ }^{*}$, LIN ZHONG $^{1 *}$, LEI GONG $^{2}$, JIAHUI WANG ${ }^{3}$, YUJIE ZHU ${ }^{1}$, WEIFENG LIU ${ }^{1}$ and JUN YANG ${ }^{1}$ \\ ${ }^{1}$ Department of Cardiology, ${ }^{2}$ Biochip Laboratory, and ${ }^{3}$ Central Laboratory, Yantai Yuhuangding Hospital, \\ Affiliated Hospital of Medical College Qingdao University, Yantai, Shandong 264000, P.R. China
}

Received February 25, 2017; Accepted September 8, 2017

DOI: $10.3892 /$ ijmm.2017.3140

\begin{abstract}
Fibroblast growth factor 21 (FGF21), as an endocrine factor, is secreted into circulation by injured cardiomyocytes. Endoplasmic reticulum (ER) stress-induced apoptosis has been proposed as an important pathophysiological mechanism for cardiomyocyte injury. However, whether the enhanced expression of FGF21 in cardiomyocytes is linked to ER stress, and the effect and underlying mechanism of FGF21 on ER stress-induced cardiomyocyte apoptosis remain unclear. In the present study, it was demonstrated that mild ER stress resulted in upregulated expression levels of FGF21 and its main receptors, as a response to cell compensation, at the induction of $\leq 5 \mu \mathrm{M}$ tunicamycin (TM). However, excessive ER stress (TM $\geq 10 \mu \mathrm{M}$ ) activated the ER stress-mediated apoptosis signaling pathways, including PKR-like ER kinase (PERK)-eukaryotic translational initiation factor $2 \alpha$ (eIF $2 \alpha)$-activating transcrip-
\end{abstract}

Correspondence to: Dr Jun Yang, Department of Cardiology, Yantai Yuhuangding Hospital, Affiliated Hospital of Medical College Qingdao University, 20 Yuhuangding East Road, Yantai, Shandong 264000, P.R. China

E-mail: yangjqh@126.com

${ }^{*}$ Contributed equally

Abbreviations: FGF21, fibroblast growth factor 21; ER, endoplasmic reticulum; UPR, unfolded protein response; PERK, PKR-like ER kinase; IRE1, inositol-requiring kinase $1 \alpha$; ATF6, activating transcription factor 6 ; eIF $2 \alpha$, eukaryotic translational initiation factor $2 \alpha$; ATF4, activating transcription factor 4 ; XBP1, X-box binding protein 1; JNK, c-Jun N-terminal kinase; CHOP, CCAAT/-enhancer-binding protein homologous protein; c-caspase-3, cleaved caspase-3; Bcl-2, B-cell lymphoma-2; Bax, Bcl-2 associated $\mathrm{X}$, apoptosis regulator; ERK, extracellular signalregulated kinases; TM, tunicamycin

Key words: fibroblast growth factor 21, apoptosis, endoplasmic reticulum stress, cardiomyocytes, fibroblast growth factor receptor 1 , extracellular signal-regulated kinase $1 / 2$ tion factor 4 (ATF4)-CCAAT/-enhancer-binding protein homologous protein (CHOP) and inositol-requiring kinase $1 \alpha$ (IRE1 $\alpha$ )-c-Jun N-terminal kinases (JNK), as well as inhibited the expression of FGF21 and its primary receptors. In addition, FGF21 overexpression provided protection against ER stress-induced cardiomyocyte injury, as evidenced by increased cell viability and reduced apoptosis. These changes were associated with the inhibition of ER stress-mediated apoptosis signaling pathways, as well as increased phosphorylation of FGFR1 and ERK1/2. However, the protective effects of overexpressed FGF21 were abolished following treatment with FGFR1 and ERK1/2 inhibitors. Thus, mild ER stress may induce the expression of FGF21 and its primary receptors in cardiomyocytes. FGF21 inhibits ER stress-induced cardiomyocyte injury as least in part via the FGFR1-ERK1/2 signaling pathway.

\section{Introduction}

Fibroblast growth factor 21 (FGF21) is a unique member of the FGF superfamily, which is predominantly expressed in the liver and exerts pleiotropic effects on metabolic processes (1). FGF21 may activate FGF receptors (FGFRs), including FGFR1-4, which all contain an intracellular tyrosine kinase domain, and FGFR1 is the predominant FGFR isotype in the heart and vessels (2). $\beta$-Klotho, as a co-receptor, is necessary for FGF21 to bind with particular FGFRs and effectively activate the FGF21 signaling pathway (3). Furthermore, among the various isoforms of FGFRs, FGFR1 has been suggested as the primary form for FGF21 in cardiomyocytes and the function of FGF21 was mediated primarily by binding to FGFR1 in a $\beta$-Klotho-dependent manner $(2,4)$. A previous study identified that FGF21 is upregulated and released into circulation by the liver tissue in response to myocardial ischemia (5). Serum levels of FGF21 are also elevated in patients with coronary artery disease, acute myocardial infarction and heart failure (6-8). Additionally, FGF21 may decrease ischemia-reperfusion injury of cardiomyocytes and improve the antioxidant capacity of endothelial cells $(9,10)$, indicating that FGF21 is a critical protective factor for the cardiovascular system. 
Accumulation of unfolded or misfolded proteins at the reticulum transmembrane causes endoplasmic reticulum (ER) stress, activating an adaptive response termed the unfolded protein response (UPR) (11). The UPR involves the PKR-like ER kinase (PERK), inositol-requiring kinase $1 \alpha$ (IRE1 $\alpha$ ) and activating transcription factor 6 (ATF6) (12), which all regulate the transcription of a variety of associated genes encoding ER chaperone proteins to clear the unfolded protein aggregation at the initial stage of ER stress. When ER stress is prolonged and/or excessive, the UPR fails to control the level of unfolded or misfolded proteins in the ER, subsequently leading to the activation of apoptotic pathways (11-13). Proteins downstream of the PERK and IRE1 signaling pathways have been identified as having pro-apoptotic roles. PERK phosphorylates eIF2 $\alpha$ to induce the translation of ATF4. ATF4 modulates a wide range of genes involved in the adaptation to ER stress and induces CCAAT/-enhancer-binding protein homologous protein (CHOP) to initiate the apoptosis signaling pathway $(14,15)$. IRE1 $\alpha$ mediates the accumulation of misfolded proteins, and induces downstream molecular X-box binding protein 1 (XBP1) and c-Jun N-terminal kinase (JNK). However, IRE1 $\alpha$-dependent apoptotic signaling occurs via phosphorylating JNK (16), which induces cell death via the induction of various proteins from the B-cell lymphoma-2 (Bcl-2) family and the activation of cysteinyl aspartate specific proteinase (caspases) (17,18).

Evidence indicates that the apoptosis induced by ER stress is regarded as a precursor to the inflammatory reaction, and is a central mechanism, which leads to the development and progression of cardiovascular diseases, including atherosclerosis, ischemic heart diseases and heart failure (19-21), which all exhibit markedly increased FGF21 expression levels. However, whether the enhanced expression level of FGF21 is associated with ER stress in cardiomyocytes, and the functional role and molecular mechanism of FGF21 in ER stress-induced myocardial apoptosis remain unknown.

\section{Materials and methods}

Reagents. Fetal bovine serum (FBS) and Dulbecco's modified Eagle's medium (DMEM) were purchased from Gibco (Thermo Fisher Scientific, Inc., Waltham, MA, USA). The rabbit anti $\beta$-Klotho (cat. no. AV53325) and mouse anti-c-myc (cat. no. M5546) antibodies were purchased from Sigma-Aldrich (Merck KGaA, Darmstadt, Germany). The rabbit anti-FGF21 (cat. no. Ab171941), rabbit anti-phosphorylated (p)-FGFR1 (cat. no. Ab59194) and rabbit anti-p-IRE1 $\alpha$ (cat. no. Ab124945) antibodies were obtained from Abcam (Cambridge, MA, USA). The rabbit anti-FGFR1 (cat. no. 9740), rabbit anti-extracellular signal-regulated kinases (ERK)1/2 (cat. no. 4695), rabbit anti-p-ERK1/2 (cat. no. 4370), rabbit antiPERK (cat. no. 3192), rabbit anti-p-PERK (cat. no. 3179), rabbit anti-ATF4 (cat. no. 11815), rabbit anti-IRE1 $\alpha$ (cat. no. 3294), rabbit anti-JNK (cat. no. 9252), rabbit anti-p-JNK (cat. no. 9251), rabbit anti-eIF2 $\alpha$ (cat. no. 5324), rabbit antip-eIF2 $\alpha$ (cat. no. 3398) and rabbit anti-cleaved-caspase-3 (c-caspase-3; cat. no. 9661) antibodies were purchased from Cell Signaling Technology, Inc. (Danvers, MA, USA). The rabbit anti-CHOP (cat. no. SC-7351) and mouse anti- $\beta$-actin (cat. no. SC-47778) antibodies were purchased from Santa Cruz Biotechnology, Inc. (Dallas, TX, USA). The rabbit anti-Bcl-2 (cat. no. BA0315) and rabbit anti-Bcl-2 associated $\mathrm{X}$ protein (cat. no. BA0412) antibodies were purchased from Wuhan Boster Biological Technology, Ltd. (Wuhan, China). Antimouse IgG (cat. no. 7076) and anti-rabbit IgG (cat. no. 7074), PD166866 and PD98059 were purchased from Cell Signaling Technology. Tunicamycin (TM) was purchased from Sangon Biotech Co., Ltd. (Shanghai, China).

Cell culture. Rat H9c2 cardiomyocytes (Chinese Academy of Sciences Cell Bank, Shanghai, China) were cultured in high glucose DMEM supplemented with 10\% (v/v) FBS and $2 \mathrm{mM}$ L-glutamine at $37^{\circ} \mathrm{C}$ under a $5 \% \mathrm{CO}_{2}$ atmosphere. For the present study $\mathrm{H} 9 \mathrm{c} 2$ cells were plated at $\sim 60 \%$ density and incubated overnight to reach $70-80 \%$ confluence at $37^{\circ} \mathrm{C}$ before experimentation.

Recombinant plasmid construction and transfection. The cDNA encoding full-length rat FGF21 (627 bp; accession no. NM_ 130752.1) was cloned and inserted into the XhoI and EcoRI restriction sites of pcDNA4/myc-his (5,151 bp) to generate the expression plasmid for rat FGF21 (pcDNA4FGF21). The pcDNA4-FGF21 plasmid was verified by special digestion analysis and DNA sequencing and transfected into cells using X-tremeGENE HP DNA Transfection reagent (Roche Diagnostics, Laval, QC, Canada) for $48 \mathrm{~h}$.

Experimental protocols. The cultured H9c2 cardiomyocytes were randomly divided into different groups. In the control group, the H9c2 cardiomyocytes were incubated under normal conditions. In the TM-treated group, the H9c2 cardiomyocytes were treated with various doses $(1,5,10,20$ and $50 \mu \mathrm{M}$ ) of TM for $24 \mathrm{~h}$. In the FGF21 overexpressiontreated group (pcDNA4-FGF21 + TM), H9c2 cardiomyocytes were transfected with pcDNA4-FGF21 plasmid for $48 \mathrm{~h}$ and subsequently exposed to TM $(10 \mu \mathrm{M})$ treatment for a further $24 \mathrm{~h}$. Inhibitor-treated groups were processed the same as the pcDNA4-FGF21 + TM group, but the cells were co-incubated with the specific FGFR1 inhibitor, PD166866 $(100 \mathrm{nM})$ or ERK1/2 inhibitor, PD98059 $(20 \mu \mathrm{M})$ for $1 \mathrm{~h}$ before treatment with the pcDNA4-FGF21 plasmid. Three experimental categories were included: i) the control and TM $(1,5,10,20$ and $50 \mu \mathrm{M})$ groups; ii) the control, TM $(10 \mu \mathrm{M})$, pcDNA4-FGF21 + TM $(10 \mu \mathrm{M})$, and pcDNA4 + TM $(10 \mu \mathrm{M})$ groups; iii) the TM $(10 \mu \mathrm{M})$, pcDNA4-FGF21 + TM $(10 \mu \mathrm{M})$, pcDNA4-FGF21 + TM $(10 \mu \mathrm{M})+$ PD166866, and pcDNA4FGF21 + TM $(10 \mu \mathrm{M})+$ PD98059 groups.

Cell viability assay. Cell viability was measured using Cell Counting Kit-8 (CCK-8, cat. no. CK04; Dojindo Laboratories, Kumamoto, Japan) according to the manufacturer's instructions. Cells were dispensed in 96-well plates at a density of $7 \times 10^{3}$ cells/well and incubated overnight at $4^{\circ} \mathrm{C}$. After the designated treatment, cells were washed in DMEM and $10 \mu \mathrm{l}$ CCK-8 solution was added to each well for a 4-h incubation. Subsequently, the optical density (OD) of each well was measured at a wavelength of $450 \mathrm{~nm}$ using a spectrophotometer (BioTek Instruments, Inc., Winooski, VT, USA). Cell viability $(\%)=\left(\mathrm{OD}_{\text {sample }}-\mathrm{OD}_{\text {blank }}\right) /\left(\mathrm{OD}_{\text {control }}-\mathrm{OD}_{\text {blank }}\right) \times 100 \%$. 
FGF21 secretion level measurement. H9c2 cells were maintained in medium containing $0.2 \%$ BSA rather than $10 \%$ FBS and treated with various concentrations of TM for $24 \mathrm{~h}$. FGF21 released in the culture medium under ER stress conditions was determined using an ELISA kit (cat. no. MF2100; R\&D Systems, Inc., Minneapolis, MN, USA) according to the manufacturer's instructions. All reactions were performed in triplicate using 96-well ELISA plates.

Terminal deoxynucleotidyl-transferase-mediated dUTP nick end labeling (TUNEL) staining. TUNEL assay was performed using a One Step TUNEL apoptosis kit (cat. no. KGA4052; Nanjing KeyGen Biotech Co., Ltd., Nanjing, China) according to the manufacturer's instruction. Cells were plated on glass coverslips at a density of $1 \times 10^{5}$ cells/well. Following treatment, the cells were fixed in $4 \%$ formaldehyde for $30 \mathrm{~min}$ and permeabilized in $0.5 \%$ Triton X-100 for $10 \mathrm{~min}$. The cells were labeled with dUTP and TDT enzymes in a humidified box at $37^{\circ} \mathrm{C}$ for $1 \mathrm{~h}$, subsequently incubated in streptavidin-fluorescein (SF) at room temperature for $30 \mathrm{~min}$ and counterstained with 4',6-diamidino-2-phenylindole for $10 \mathrm{~min}$ before observation under a laser scanning confocal microscope (Bio-Rad Laboratories, Inc., Hercules, CA, USA). TUNEL-positive cells exhibited a bright green fluorescence and $>5$ non-overlapping fields in each group were quantified.

Protein extraction and western blot analysis. Total protein was extracted according to the methods used by Kurian et al (22). Protein concentration was determined using a BCA protein assay kit (cat. no. P0012; Beyotime Institute of Biotechnology, Haimen, China) with BSA as standard. Total proteins (30 $\mu \mathrm{g})$ were fractionated by $8-12 \%$ sodium dodecyl sulfate-polyacrylamide gel electrophoresis (SDS-PAGE) and transferred onto a polyvinylidene difluoride (PVDF) membrane (Immobilon P; Millipore, Bedford, MA, USA) at $90 \mathrm{~V}$ constant for $100 \mathrm{~min}$. The blots were blocked with $5 \%$ BSA for $1 \mathrm{~h}$ and the membranes were incubated with antibodies diluted in 5\% BSA at $4^{\circ} \mathrm{C}$ overnight. The primary antibodies used in the present study were as follows: FGF21 (1:500 dilution), FGFR1 (1:250 dilution), p-FGFR1 (1:200 dilution), $\beta$-Klotho (1:500 dilution), c-myc (1:1,000 dilution), ERK1/2 (1:3,000 dilution), p-ERK1/2 (1:3,000 dilution), PERK (1:1,000 dilution), p-PERK (1:1,000 dilution), eIF2 $\alpha$ (1:750 dilution), p-eIF2 $\alpha$ (1:1,000 dilution), ATF4 (1:5,000 dilution), CHOP (1:1,000 dilution), IRE1 $\alpha$ (1:1,000 dilution), p-IRE1 $\alpha$ (1:1,000 dilution), JNK(1:1,000 dilution), p-JNK (1:1,000 dilution), Bcl-2 (1:200 dilution), Bax (1:200 dilution), c-caspase-3 (1:200 dilution) and $\beta$-actin (1:2,000 dilution).

After washing three times with $0.1 \%$ Tris-buffered saline with Tween-20 (TBST) and incubating with HRP-conjugated secondary antibodies [anti-mouse IgG (1:5,000 dilution) and anti-rabbit $\operatorname{IgG}$ (1:5,000 dilution)] for $1 \mathrm{~h}$ at room temperature, specific bands were visualized by enhanced chemiluminescence detection (cat. no. 34077; Thermo Fisher Scientific, Inc.). Bands were analyzed by the ImageJ software (National Institutes of Health, Bethesda, MD, USA). Each experiment was repeated at least three times.

Statistical analysis. Statistical analysis was performed using the SPSS 19.0 software (SPSS, Inc., Chicago, IL, USA).
Significant differences between the groups were analyzed by the unpaired Student's t-test or the one-way ANOVA and $\mathrm{P}<0.05$ was considered to indicate a statistically significant difference. Data were presented as means \pm standard deviation.

\section{Results}

TM inhibits the viability of H9c2 cells and induces ER stressassociated signaling pathways. To establish whether the ER stress model had successfully been established by TM, H9c2 cells were treated with increased concentrations $(1,5,10$, 20 and $50 \mu \mathrm{M})$ of TM for $24 \mathrm{~h}$. Initially, the effect of TM on the cell viability of H9c2 cells was examined. As shown in Fig. 1A, TM up to $5 \mu \mathrm{M}$ did not significantly reduce the viability of $\mathrm{H} 9 \mathrm{c} 2$ cells, but TM became cytotoxic to $\mathrm{H} 9 \mathrm{c} 2$ cells at a dose of $>10 \mu \mathrm{M}$ and cell viability reduced with increased concentrations of TM. Thus, $10 \mu \mathrm{M}$ TM was used in subsequent experiments for induction of ER stress. In addition, the PERK-eIF2 $\alpha$-AFT4-CHOP signaling pathway and IRE1 $\alpha-J N K$ mediated signaling pathway are well known ER stress-associated signaling pathways for inducing apoptosis. Following treatment with TM up to $5 \mu \mathrm{M}$, mild ER stress partly activated these signaling pathways, which was evidenced by induction of p-PERK, p-eIF2 $\alpha$, ATF4 and CHOP in a dosedependent manner (Fig. 1B). In addition, mild ER stress caused upregulated expression of p-IRE1 $\alpha, \mathrm{p}$-JNK, c-caspase-3 and Bax while it reduced the level of anti-apoptosis protein, $\mathrm{Bcl}-2$ (Fig. 1C). However, at a high concentration $(\geq 10 \mu \mathrm{M})$ of $\mathrm{TM}$, this increasing level reached a maximum at a concentration of $10 \mu \mathrm{M}$ TM and plateaued with the increasing concentration, this indicated that excessive ER stress ( $\geq 10 \mu \mathrm{M}$ TM) fully activated ER stress-associated signaling pathways to induce further cell apoptosis.

Mild ER stress elevates the expression levels of FGF21 and its main receptor in $H 9 c 2$ cells. TM treatment significantly induced endogenous FGF21, p-FGFR1 and $\beta$-Klotho expression levels in H9c2 cells (Fig. 2A). Furthermore, increased levels of secreted FGF21 protein were detected from the culture medium of TM-treated H9c2 cells (Fig. 2B). However, the elevated expression level and secretion of these proteins were not positively correlated with the degree of ER stressinduced cell damage. Notably, in mild ER stress ( $\leq 5 \mu \mathrm{M}$ TM), the protein expression level of FGF21, p-FGFR1 and $\beta$-Klotho increased and peaked at concentration of $5 \mu \mathrm{M}$ TM. However, in excessive ER stress ( $\geq 10 \mu \mathrm{M}$ TM), the expression level of FGF21 and its main receptors decreased gradually with aggravation of the ER stress level. These results indicated that the elevated expression of FGF21 and its main receptors may be a compensatory response to injury in mild ER stress.

FGF21 prevents the ER stress-induced apoptosis of $\mathrm{H} 9 \mathrm{c} 2$ cells. FGF21 was overexpressed from the pcDNA4-FGF21 eukaryotic expression vector in $\mathrm{H} 9 \mathrm{c} 2$ cells (Fig. 3A and B). To demonstrate the cardioprotective effect of FGF21 on ER stress-induced cardiomyocyte injury, H9c2 cells overexpressing FGF21 were exposed to TM $(10 \mu \mathrm{M})$ treatment for 24 h. The result demonstrated that FGF21 overexpression significantly reversed the decreased cell viability caused by TM treatment, indicating that FGF21 protected H9c2 cells 

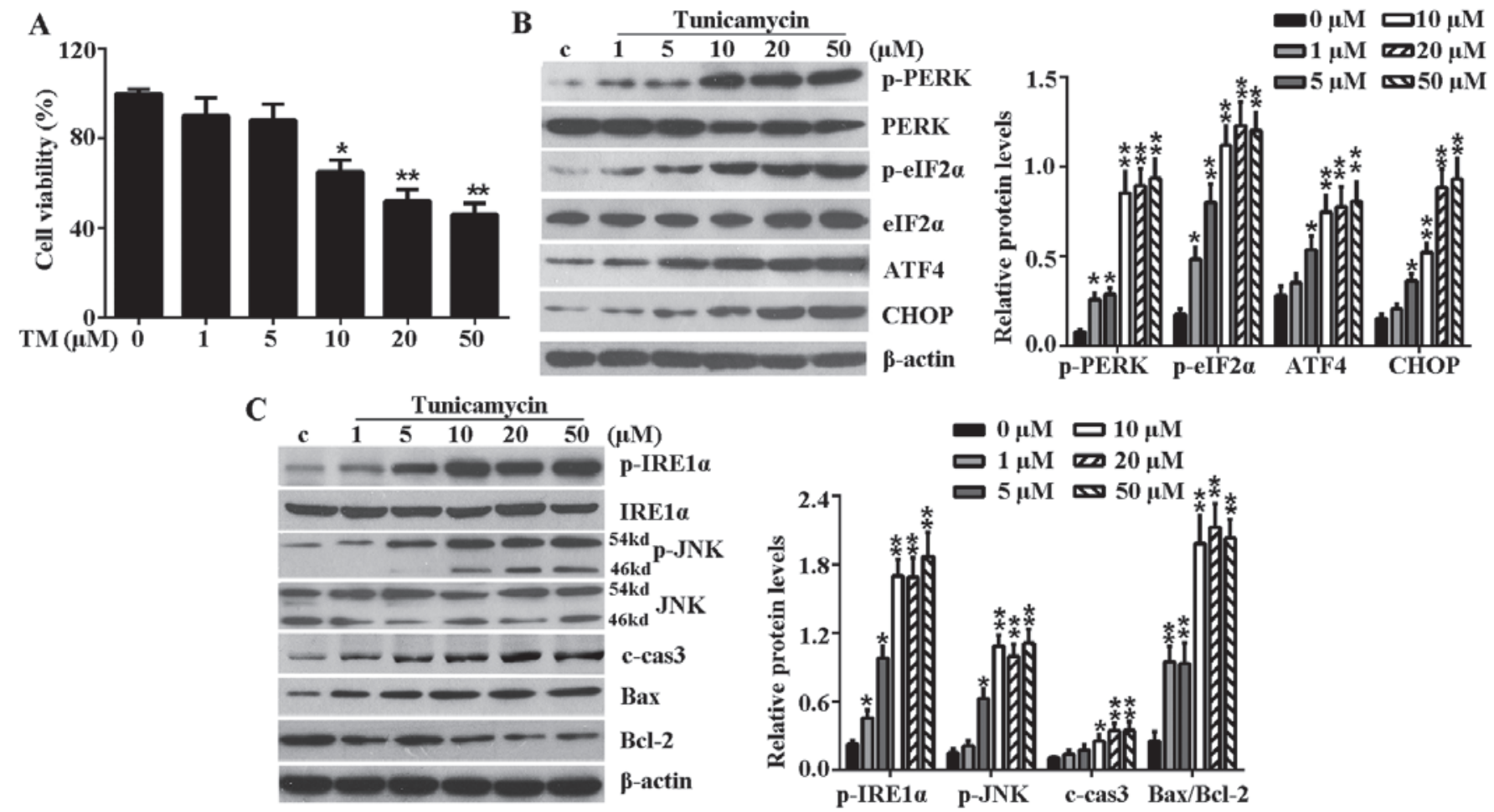

Figure 1. TM inhibited the cell viability of H9c2 cells and induced ER stress-associated signaling pathways. H9c2 cells were treated with various concentrations $(1,5,10,20$ and $50 \mu \mathrm{M})$ of TM for $24 \mathrm{~h}$. (A) Cell viability was detected by Cell Counting Kit- 8 assay. (B) Western blot analysis of the expression levels of PERK-eIF2 $\alpha$-ATF4-CHOP signaling pathway-associated key proteins, including p-PERK, PERK, p-eIF2 $\alpha$, eIF2 $\alpha$, ATF4 and CHOP. (C) Western blot analysis of IRE1 $\alpha$-JNK signaling pathway-associated proteins, including p-IRE1 $\alpha$, IRE1 $\alpha$, p-JNK, JNK, c-caspase-3, Bax and Bcl-2. $\beta$-actin served as a loading control. Error bars demonstrate the means. ${ }^{*} \mathrm{P}<0.05$ or ${ }^{* *} \mathrm{P}<0.01$ vs. control. TM, tunicamycin; ER, endoplasmic reticulum; PERK, PKR-like ER kinase; eIF2 $\alpha$, eukaryotic translational initiation factor 2 $\alpha$; ATF4, activating transcription factor 4; CHOP, CCAAT/-enhancer-binding protein homologous protein; p, phosphorylated; JNK, c-Jun N-terminal kinase; IRE1 $\alpha$, inositol-requiring kinase 1 $\alpha$; c-cas3, cleaved caspase-3; Bax, Bcl-2 associated X protein; Bcl-2, B-cell lymphoma-2; c, control.
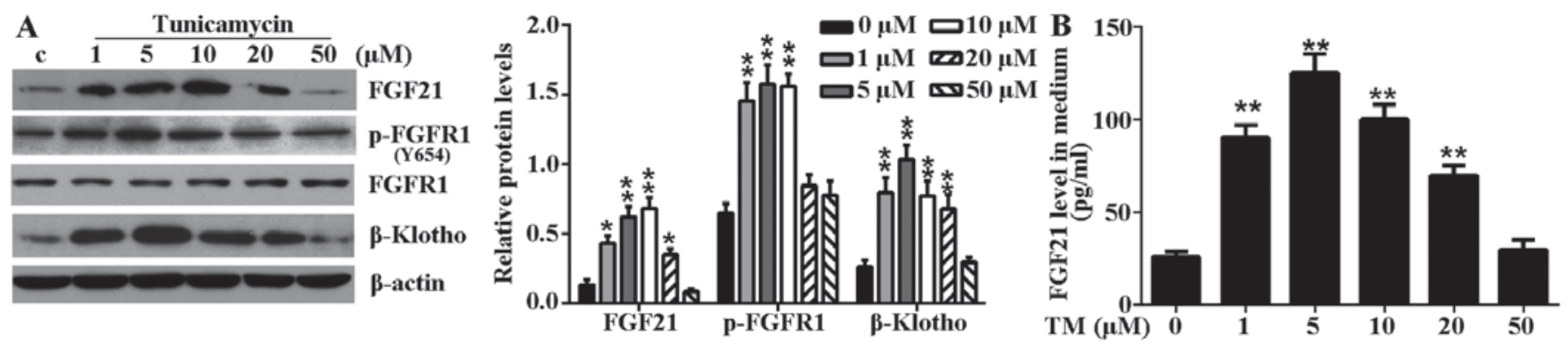

Figure 2. TM-induced ER stress induced FGF21 expression and increased circulating FGF21 expression levels in H9c2 cells. (A) H9c2 cells were treated with various concentrations $(1,5,10,20$ and $50 \mu \mathrm{M})$ of TM for $24 \mathrm{~h}$. The expression levels of FGF21, FGFR1, p-FGFR1 and $\beta$-Klotho in H9c2 cells in response to TM-induced ER stress were evaluated by western blot analysis. $\beta$-actin served as a loading control. (B) Secreted FGF21 levels in culture medium following ER stress stimulation were measured by ELISA assay. ${ }^{*} \mathrm{P}<0.05$ or ${ }^{* * *} \mathrm{P}<0.01$ vs. control. TM, tunicamycin; ER, endoplasmic reticulum; FGF21, fibroblast growth factor 21; FGFR1, fibroblast growth factor receptor 1; p, phosphorylated; c, control.

from ER stress-mediated cell death (Fig. 3C). In addition, the TUNEL assay indicated that ER stress-induced apoptosis was significantly reduced by FGF21 overexpression (Fig. 3D).

FGF21 inhibits ER stress-related signaling pathways. The effects of FGF21 overexpression on the key proteins of the PERK-eIF2 $\alpha$-AFT4-CHOP signaling pathway and IRE1 $\alpha-J N K$ signaling pathway were determined in $\mathrm{H} 9 \mathrm{c} 2$ cells under ER stress. Western blotting revealed that the expression level of p-PERK, p-eIF2 $\alpha$, ATF4 and CHOP were markedly increased following $24 \mathrm{~h}$ of TM treatment compared with the untreated cells. However, FGF21 overexpression significantly reduced these inductions (Fig. 4A). In addition, as shown in Fig. 4B, the upregulation of p-IRE1 $\alpha$, p-JNK, c-caspase-3, Bax and Bcl-2 were markedly reversed by FGF21 overexpression, indicating that FGF21 overexpression inhibited these ER stress evoked pro-apoptotic signaling events in $\mathrm{H} 9 \mathrm{c} 2$ cells.

FGF21 inhibits ER stress-induced apoptosis via FGFR1 and ERK1/2 phosphorylation. The expression levels of FGF21, p-FGFR1 and p-ERK1/2 significantly increased in H9c2 cells transfected with pcDNA4-FGF21 compared with those transfected with an empty vector (Fig. 5A), indicating that FGF21 


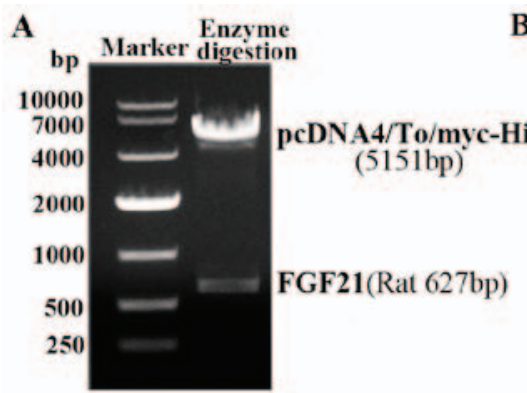

PeDNA4 PeDNA4FGF21

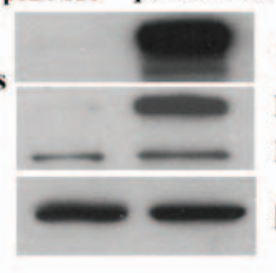

c-myc

FGF21-myc FGF21

$\beta$-actin
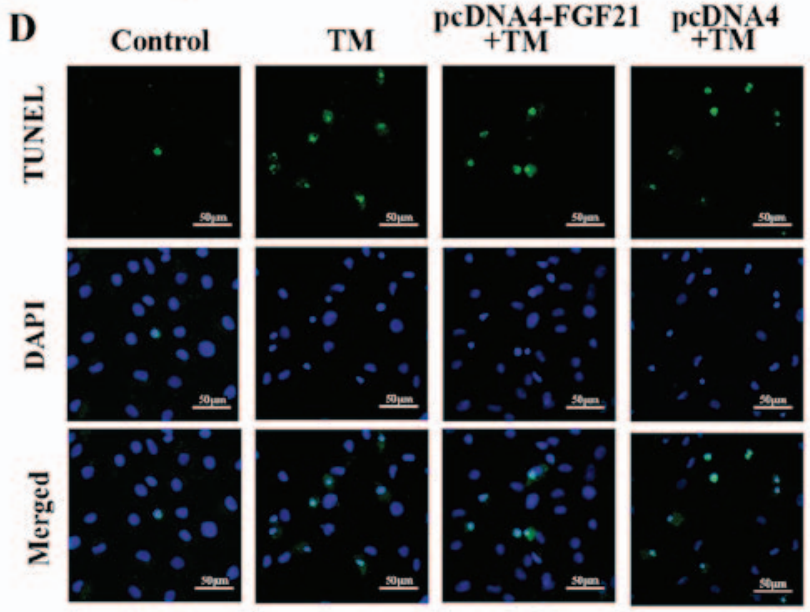

Figure 3. Overexpressed FGF21 protected H9c2 cells from ER stress-induced apoptosis. (A) The recombinant plasmid pcDNA4-FGF21 was constructed by pcDNA4/To/myc-his (5,151 bp) and full-length rat FGF21 (627 bp), confirmed by agarose gel electrophoresis and gene sequencing. (B) Western blot analysis demonstrating the overexpression of myc-labelled FGF21 protein from the transfected pcDNA4-FGF21 plasmid in H9c2 cells using a c-myc tag and FGF21 antibodies. (C) H9c2 cells were transfected with pcDNA4-FGF21 plasmid for $48 \mathrm{~h}$, and exposed to TM (10 $\mu \mathrm{M})$ treatment for a further $24 \mathrm{~h}$, the cell viability was detected by Cell Counting Kit-8 assay. (D) Fluorescence microscopy of TUNEL staining of H9c2 cells. Cell nucleus was stained with DAPI (blue). Scale bar, $50 \mu \mathrm{m}$. The percentage of apoptotic cells in each group was calculated by counting the condensed nuclei. ${ }^{* *} \mathrm{P}<0.01 \mathrm{vs}$. control, ${ }^{*} \mathrm{P}<0.05 \mathrm{vs}$. TM-treated cells, ${ }^{\#} \mathrm{P}<0.05$ vs. pcDNA4 + TM group. FGF21, fibroblast growth factor 21; ER, endoplasmic reticulum; TM, tunicamycin; TUNEL, terminal deoxynucleotidyltransferase-mediated dUTP nick end labeling; DAPI, 4',6-diamidino-2-phenylindole.
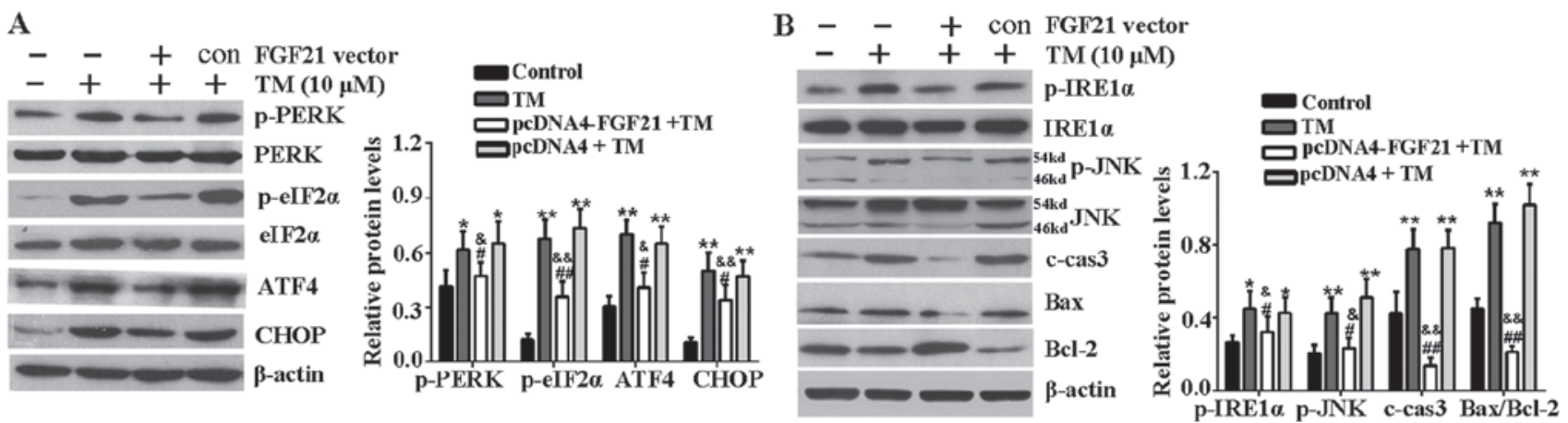

Figure 4. FGF21 inhibited ER stress-mediated apoptosis signaling pathways. (A) H9c2 cells were treated with TM (10 $\mu \mathrm{M})$ following transfection with pcDNA4-FGF21 plasmid for $48 \mathrm{~h}$, the expression of PERK-eIF2 $\alpha$-ATF4-CHOP signaling pathway-associated key proteins, including p-PERK, PERK, p-eIF2 $\alpha$, eIF $2 \alpha$, ATF4 and CHOP were analyzed by western blotting. (B) Western blot analysis of IRE1 $\alpha$-JNK signaling pathway-associated proteins, including p-IRE1 $\alpha$, IRE1 $\alpha$, p-JNK, JNK, c-caspase-3, Bax and Bcl-2. $\beta$-actin served as a loading control. Error bars represent standard deviation. "P $<0.05$ or ${ }^{* *} \mathrm{P}<0.01$ vs. control, ${ }^{\&} \mathrm{P}<0.05$ or ${ }^{\& \&} \mathrm{P}<0.01$ vs. TM group, ${ }^{\#} \mathrm{P}<0.05$ or ${ }^{\# \#} \mathrm{P}<0.01$ vs. pcDNA4 + TM group. FGF21, fibroblast growth factor 21 ; ER, endoplasmic reticulum; TM, tunicamycin; PERK, PKR-like ER kinase; eIF2 $\alpha$, eukaryotic translational initiation factor 2 $\alpha$; ATF4, activating transcription factor 4; CHOP, CCAAT/-enhancer-binding protein homologous protein; p, phosphorylated; JNK, c-Jun N-terminal kinase; IRE1 $\alpha$, inositol-requiring kinase 1 $\alpha$; c-cas3, cleaved caspase-3; Bax, Bcl-2 associated X protein; Bcl-2, B-cell lymphoma-2; c, control.

may positively regulate the expression level of p-FGFR1 and p-ERK1/2 to attenuate ER stress-induced injury. To determine whether FGF21-induced FGFR1 and ERK1/2 activation is responsible for its cardioprotective effect, H9c2 cells were treated with FGFR1 inhibitor, PD166866 or ERK1/2 inhibitor, PD98059 before being transfected with pcDNA4-FGF21 and the addition of TM. The results demonstrate that the improvement of cell viability mediated by FGF21 overexpression was significantly reduced (Fig. 5B). In addition, the TUNEL assay indicated that FGFR1 and ERK1/2 inhibitors attenuated the FGF21 overexpression-mediated anti-apoptotic effect on ER stress injury (Fig. 5C). 


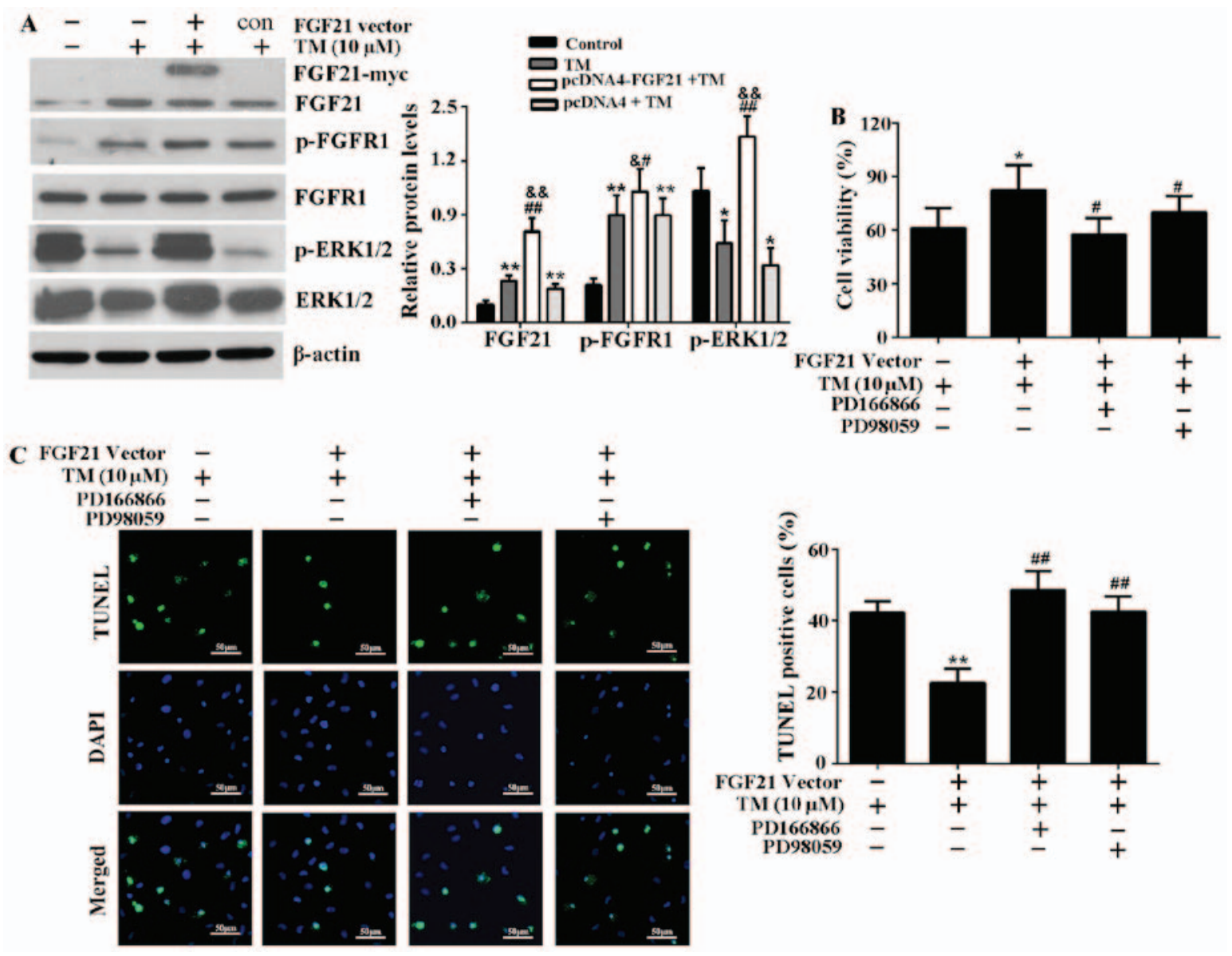

Figure 5. FGF21 overexpression activated FGFR1 and ERK1/2 in H9c2 cells, and the inhibitors of FGFR1 and ERK1/2 attenuate cardioprotective effect of FGF21 on ER stress injury. (A) Subsequent to transfection with the pcDNA4-FGF21 plasmid, the expression levels of FGF21, p-FGFR1, FGFR1, p-ERK1/2 and ERK1/2 were analyzed by western blotting and their relative protein expression levels were compared. ${ }^{\text {}} \mathrm{P}<0.05$ or ${ }^{* *} \mathrm{P}<0.01$ vs. control, ${ }^{\circledR} \mathrm{P}<0.05$ or ${ }^{\& \&} \mathrm{P}<0.01 \mathrm{vs}$. TM group, ${ }^{\#} \mathrm{P}<0.05$ or ${ }^{\# \#} \mathrm{P}<0.01$ vs. pcDNA4 + TM group. (B) H9c2 cells were pretreated with the specific FGFR1 inhibitor, PD166866 (100 nM) or ERK1/2 inhibitor, PD98059 $(20 \mu \mathrm{M})$ for $1 \mathrm{~h}$, then transfected with pcDNA4-FGF21 plasmids for $48 \mathrm{~h}$. TM $(10 \mu \mathrm{M})$ was added to the media and incubated at $37^{\circ} \mathrm{C}$ for $24 \mathrm{~h}$. The cell viability was evaluated by Cell Counting Kit- 8 assay. ${ }^{*} \mathrm{P}<0.05$ vs. TM group, ${ }^{\prime \prime} \mathrm{P}<0.05$ vs. pcDNA4-FGF21 + TM group. (C) Fluorescence microscopy of TUNEL staining of H9c2 cells. The cell nucleus was stained with DAPI (blue). Scale bar, $50 \mu \mathrm{m}$. The percentage of apoptotic cells in each group was calculated by counting the condensed nuclei. ${ }^{* *} \mathrm{P}<0.05$ vs. TM group, ${ }^{\# \#} \mathrm{P}<0.05$ vs. pcDNA4-FGF2 $1+\mathrm{TM}$ group. FGF21, fibroblast growth factor 21; FGFR1, fibroblast growth factor receptor 1; ERK, extracellular signal-regulated kinases; TM, tunicamycin; ER, endoplasmic reticulum; p, phosphorylated; TUNEL, terminal deoxynucleotidyl-transferase-mediated dUTP nick end labeling; c, control.

FGFR1 and ERK1/2 inhibitors attenuate FGF21-mediated inhibition of ER stress-associated signaling pathways. Subsequently the effects of FGFR1 and ERK1/2 inhibitors on the activation of FGFR1 and ERK1/2, and the inhibition of ER stress-associated signaling pathways induced by FGF21 overexpression were investigated. The results indicated that phosphorylation of FGFR1 and ERK1/2 were stimulated by FGF21 overexpression, and FGFR1 inhibitor, PD166866 attenuated the stimulated effect of FGF21 on phosphorylated ERK1/2 (Fig. 6A), indicating that ERK1/2 should be activated during the phosphorylation of FGFR1, as stimulated by FGF21. Furthermore, the decrease of key proteins associated with the PERK-eIF2 $\alpha$-ATF4-CHOP signaling pathway, including p-PERK, p-eIF2 $\alpha$, ATF4 and CHOP mediated by FGF21 overexpression were reversed following treatment with FGFR1 and ERK1/2 inhibitors (Fig. 6B). In addition, FGFR1 and ERK1/2 inhibitors reversed the reduced expression level of p-IRE1 $\alpha, \mathrm{p}$-JNK, c-caspase-3 and Bax, and the increased expression level of Bcl-2 as a result of FGF21 overexpression (Fig. 6C). Therefore, the inhibitory effect of FGF21 on the ER stress-associated signaling pathways was at least in part via the FGFR1-ERK1/2 signaling pathway.

\section{Discussion}

In the present study, ER stress was demonstrated to induce the expression of FGF21 and its main receptors in rat $\mathrm{H} 9 \mathrm{c} 2$ cardiomyocytes, although the enhanced expression levels were not positively correlated with the degree of ER stress. A recent study found that FGF21, as a target gene of ATF4, was induced by ER stress via the PERK-eIF2 $\alpha$-ATF4 signaling pathway in mouse hepatocytes (23). Furthermore, ER stress caused an upregulation of hepatic $\beta$-Klotho via an ATF4dependent signaling pathway (24). Therefore, the present study concluded that in mild ER stress $(\leq 5 \mu \mathrm{M}$ TM), the elevated expression level of FGF21 and its main receptors may be due to the partly activated PERK-eIF2 $\alpha$-ATF4 signaling pathway. In the early stage of ER stress, UPR is an important feature to protect cells against environmental interference, through a compensatory mechanism, to reduce cell damage and maintain 

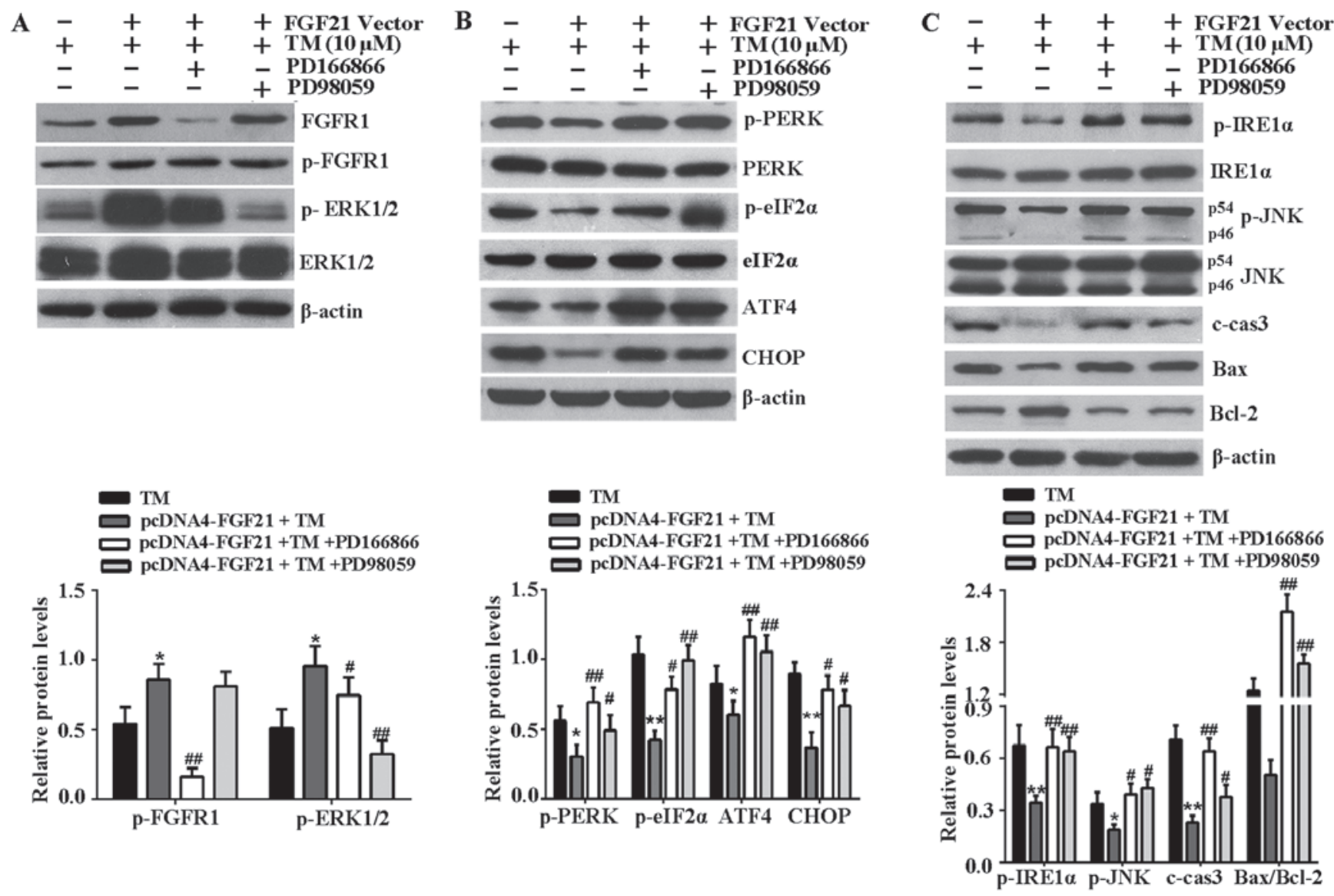

Figure 6. FGFR1 and ERK1/2 inhibitors attenuate FGF21-mediated inhibition of ER stress-induced apoptosis signaling pathways. (A) To confirm the effects of PD166866 and PD98059 on the activation of FGFR1 and ERK1/2, the cell lysates were analyzed by western blotting to detect the expression levels of p-ERK1/2, ERK1/2, p-FGFR1 and FGFR1 in H9c2 cells. (B) Western blotting was performed to detect the expression levels of the PERK-eIF2 $\alpha$-ATF4-CHOP signaling pathway-associated key proteins, including p-PERK, PERK, p-eIF2 $\alpha$, eIF2 $\alpha$, ATF4 and CHOP. (C) Western blot analysis of IRE1 $\alpha$-JNK signaling pathway-associated proteins, including p-IRE1 $\alpha$, IRE1 $\alpha$, p-JNK, JNK, c-caspase-3, Bax and Bcl-2. $\beta$-actin served as a loading control. "P<0.05 or ${ }^{* * *} \mathrm{P}<0.01$ vs. TM group, ${ }^{\#} \mathrm{P}<0.05$ or ${ }^{\# \#} \mathrm{P}<0.01$ vs. pcDNA4-FGF21 + TM group. FGFR1, fibroblast growth factor receptor 1; ERK, extracellular signal-regulated kinases; FGF21, fibroblast growth factor 21; TM, tunicamycin; ER, endoplasmic reticulum; p, phosphorylated; PERK, PKR-like ER kinase; eIF2 $\alpha$, eukaryotic translational initiation factor $2 \alpha$; ATF4, activating transcription factor 4; CHOP, CCAAT/-enhancer-binding protein homologous protein; p, phosphorylated; JNK, c-Jun N-terminal kinase; IRE1 $\alpha$, inositol-requiring kinase 1 $\alpha$; c-cas3, cleaved caspase-3; Bax, Bcl-2 associated X protein; Bcl-2, B-cell lymphoma-2; c, control.

ER homeostasis $(11,12)$, so the induced FGF21 expression and secretion may be the case of a native compensatory mechanism in response to ER stress.

However, in the case of excessive ER stress or an inadequate adaptation to stress, apoptotic signaling pathways are activated. In excessive ER stress ( $\geq 10 \mu \mathrm{M}$ TM), the PERKeIF2 $\alpha$-ATF4 and IRE1a-JNK signaling pathways were fully activated to induce a large number of proapoptotic proteins, including CHOP, caspase-3 and Bax and decrease the levels of anti-apoptosis protein, Bcl-2. Previous reports have shown that the activation of JNK induces apoptosis by activation of caspase- 3 and Bax, and inhibition of Bcl-2 in ER stress $(18,25,26)$. CHOP, as the key protein of ER stress to induced cell apoptosis, could predominantly be induced by the PERK-eIF2 $\alpha$-ATF4 signaling pathway (27). The expression levels of FGF21 and CHOP are dependent on the ATF4 signaling pathway, although more CHOP was induced by ATF4 in excessive ER stress, which may lead to competitive inhibition of FGF21, which may explain the reduced FGF21 at $\mathrm{TM}$ concentrations $>10 \mu \mathrm{M}$. Thus, the present study concluded that FGF21, as a cardioprotective cytokine, is expressed and secreted by cardiomyocytes in response to ER stress injury.
To further investigate the effect of FGF21 on ER stress injury, endogenous FGF21 expression was increased in H9c2 cells. The results demonstrated that FGF21 overexpression alleviated TM-induced ER stress and apoptosis in cardiomyocytes by interfering with the PERK-eIF2 $\alpha$-ATF4-CHOP and IREla-JNK apoptotic pathways, which in turn suppressed the activity of pro-apoptotic cytokines, thereby reducing cardiomyocyte apoptosis and supporting survival. Therefore, in the context of ER stress, the anti-apoptotic action of FGF21 may prolong the phase in which ER stress is resolved before apoptotic pathways are activated.

The present result demonstrated that the FGF21 overexpression stimulated tyrosine phosphorylation of FGFR1 in H9c2 cells, this result was consistent with a previous study reporting that the mRNA expression levels of FGFR1 and $\beta$-Klotho were induced by FGF21 overexpression (28). Recent studies have demonstrated that FGF21 exerts its biological actions via transcriptional activation of FGFRs $(1,2,4)$. FGF21 binds with high affinity and activates only FGFR1- $\beta$-Klotho, which, as a receptor complex, may regulate the function of FGF21. As a result of FGF21 overexpression, the increased expression level of p-FGFR1 is induced to meet the need 
of FGF21 in alleviating cardiomyocyte injury and restoring cardiac function.

In the present study, the phosphorylation of ERK1/2 was significantly induced by FGF21 overexpression, indicating that FGF21 may activate the ERK1/2 signaling pathway. ERK1/2, a member of the mitogen-activated protein kinases family, mediates cell proliferation and survival. Previous studies demonstrated that ERK1/2 protects the myocardium from ischemic injury (29,30). Yang et al (31) identified that FGF19 and FGF21 bind to the integrative FGFR1- $\beta$-Klotho complex and activate downstream ERK1/2 signaling in 3T3L1 adipocytes. Certain studies have confirmed that FGF21 reduces cell apoptosis via activation of the ERK1/2 signaling pathway $(15,32-34)$. Combining previous studies and the current results, the present study speculated that the protective effect of FGF21 on ER stress-induced myocardial injury was potentially via the FGFR $1 / \beta$-Klotho-ERK $1 / 2$ signaling pathways. In order to investigate this hypothesis, H9c2 cells were treated with FGFR1 and ERK1/2 inhibitors, and it was found that the anti-apoptotic effect mediated by FGF21 overexpression was attenuated, and the inhibition of FGF21 overexpression-mediated ER stress-associated signaling pathways was reversed. Furthermore, the FGFR1 inhibitor attenuated the stimulated effect of FGF21 on p-ERK1/2, indicating that the ERK1/2 signaling pathway may be controlled by the phosphorylation of FGFR1. Collectively, these results indicate that the protective effect of FGF21 on ER stress-induced myocardial injury, at least in part, is mediated by FGFR1-ERK1/2 signaling pathways.

In conclusion, the present study confirmed the elevated expression level of FGF21/FGFR1/ $\beta$-Klotho in a mild ER stress model of rat cardiomyocytes, and that FGF21 exerted a cardio-protective role against ER stress-induced cell apoptosis at least in part via activation of the FGFR1-ERK1/2 signaling pathway. These findings indicate that FGF21 therapeutic interventions may present a novel approach to preventing ER stress injury and treating cardiovascular diseases. Further studies investigating the pro-survival role of FGF21 in the context of cellular stress using FGF21 knock out mice may provide a deeper understanding of this protein.

\section{Acknowledgments}

The present study was supported by the National Natural Science Foundation of China (grant no. 81571636), the Natural Science Foundation of Shangdong, China (grant no. ZR2015HM058), the Yantai Project of Science and Technology Development Plan (grant nos. 2013WS224 and 2015WS021), the Yantai Yuhuangding Hospital Youth Scientist Research Foundation (grant no. 201526), and the Research Award Fund for Outstanding Young and Middle-aged Scientists of Shandong Province (grant no. BS2013SW043). All experiments were performed at the Shandong Research Center of Stem Cell Engineering, Yantai Yuhuangding Hospital (Yantai, China).

\section{References}

1. Kharitonenkov A, Shiyanova TL, Koester A, Ford AM, Micanovic R, Galbreath EJ, Sandusky GE, Hammond LJ, Moyers JS, Owens RA, et al: FGF-21 as a novel metabolic regulator. J Clin Invest 115: 1627-1635, 2005.
2. Fon Tacer K, Bookout AL, Ding X, Kurosu H, John GB, Wang L, Goetz R, Mohammadi M, Kuro-o M, Mangelsdorf DJ, et al: Research resource: Comprehensive expression atlas of the fibroblast growth factor system in adult mouse. Mol Endocrinol 24: 2050-2064, 2010.

3. Ogawa Y, Kurosu H, Yamamoto M, Nandi A, Rosenblatt KP, Goetz R, Eliseenkova AV, Mohammadi M and Kuro-o M: BetaKlotho is required for metabolic activity of fibroblast growth factor 21. Proc Natl Acad Sci USA 104: 7432-7437, 2007.

4. Foltz IN, Hu S, King C, Wu X, Yang C, Wang W, Weiszmann J, Stevens J, Chen JS, Nuanmanee N, et al: Treating diabetes and obesity with an FGF21-mimetic antibody activating the $\beta$ Klotho/ FGFR1c receptor complex. Sci Transl Med 4: 162ra153, 2012.

5. Liu SQ, Roberts D, Kharitonenkov A, Zhang B, Hanson SM, $\mathrm{Li}$ YC, Zhang LQ and Wu YH: Endocrine protection of ischemic myocardium by FGF21 from the liver and adipose tissue. Sci Rep 3: 2767,2013

6. Shen Y, Ma X, Zhou J, Pan X, Hao Y, Zhou M, Lu Z, Gao M, Bao Y and Jia W: Additive relationship between serum fibroblast growth factor 21 level and coronary artery disease. Cardiovasc Diabetol 12: 124, 2013.

7. Zhang W, Chu S, Ding W and Wang F: Serum level of fibroblast growth factor 21 is independently associated with acute myocardial infarction. PLoS One 10: e0129791, 2015.

8. Di Lisa F and Itoh N: Cardiac Fgf21 synthesis and release: An autocrine loop for boosting up antioxidant defenses in failing hearts. Cardiovasc Res 106: 1-3, 2015.

9. Lü Y, Liu JH, Zhang LK, Du J, Zeng XJ, Hao G, Huang J, Zhao DH, Wang GZ and Zhang YC: Fibroblast growth factor 21 as a possible endogenous factor inhibits apoptosis in cardiac endothelial cells. Chin Med J (Engl) 123: 3417-3421, 2010.

10. Cong WT, Ling J, Tian HS, Ling R, Wang Y, Huang BB, Zhao T, Duan YM, Jin LT and Li XK: Proteomic study on the protective mechanism of fibroblast growth factor 21 to ischemia-reperfusion injury. Can J Physiol Pharmacol 91: 973-984, 2013.

11. Walter P and Ron D: The unfolded protein response: From stress pathway to homeostatic regulation. Science 334: 1081-1086, 2011.

12. Faitova J, Krekac D, Hrstka R and Vojtesek B: Endoplasmic reticulum stress and apoptosis. Cell Mol Biol Lett 11: 488-505, 2006.

13. Chaudhari N, Talwar P, Parimisetty A, Lefebvre d'Hellencourt C and Ravanan P: A molecular web: Endoplasmic reticulum stress, inflammation, and oxidative stress. Front Cell Neurosci 8: 213, 2014.

14. Lee WK, Chakraborty PK, Roussa E, Wolff NA and Thévenod F: ERK1/2-dependent bestrophin-3 expression prevents ER-stressinduced cell death in renal epithelial cells by reducing CHOP. Biochim Biophys Acta 1823: 1864-1876, 2012.

15. Oyadomari S and Mori M: Roles of CHOP/GADD153 in endoplasmic reticulum stress. Cell Death Differ 11: 381-389, 2004.

16. Urano F, Wang X, Bertolotti A, Zhang Y, Chung P, Harding HP and Ron D: Coupling of stress in the ER to activation of JNK protein kinases by transmembrane protein kinase IRE1. Science 287: 664-666, 2000.

17. Brozzi F, Gerlo S, Grieco FA, Juusola M, Balhuizen A, Lievens S, Gysemans C, Bugliani M, Mathieu C, Marchetti P, et al: Ubiquitin D regulates IRE1 $\alpha / \mathrm{c}$-Jun $\mathrm{N}$-terminal kinase (JNK) protein-dependent apoptosis in pancreatic beta cells. J Biol Chem 291: 12040-12056, 2016.

18. Joo H, Lee HJ, Shin EA, Kim H, Seo KH, Baek NI, Kim B and Kim SH: c-Jun N-terminal kinase-dependent endoplasmic reticulum stress pathway is critically involved in arjunic acid Induced apoptosis in non-small cell lung cancer cells. Phytother Res 30: 596-603, 2016.

19. Minamino T and Kitakaze M: ER stress in cardiovascular disease. J Mol Cell Cardiol 48: 1105-1110, 2010.

20. Brahma MK, Adam RC, Pollak NM, Jaeger D, Zierler KA, Pöcher N, Schreiber R, Romauch M, Moustafa T, Eder S, et al: Fibroblast growth factor 21 is induced upon cardiac stress and alters cardiac lipid homeostasis. J Lipid Res 55: 2229-2241, 2014.

21. Hotamisligil GS: Endoplasmic reticulum stress and atherosclerosis. Nat Med 16: 396-399, 2010.

22. Kurian MV, Hamilton L, Keeven J, Mehl P and Mullins JM: Enhanced cell survival and diminished apoptotic response to simulated ischemia-reperfusion in $\mathrm{H} 9 \mathrm{c} 2$ cells by magnetic field preconditioning. Apoptosis 17: 1182-1196, 2012.

23. Schaap FG, Kremer AE, Lamers WH, Jansen PL and Gaemers IC: Fibroblast growth factor 21 is induced by endoplasmic reticulum stress. Biochimie 95: 692-699, 2013. 
24. Dong K, Li H, Zhang M, Jiang S, Chen S, Zhou J, Dai Z, Fang Q and Jia W: Endoplasmic reticulum stress induces up-regulation of hepatic $\beta$-Klotho expression through ATF4 signaling pathway. Biochem Biophys Res Commun 459: 300-305, 2015.

25. Zhang C, Lu T, Wang GD, Ma C and Zhou YF: Costunolide, an active sesquiterpene lactone, induced apoptosis via ROS-mediated ER stress and JNK pathway in human U2OS cells. Biomed Pharmacother 80: 253-259, 2016.

26. Mhaidat NM, Thorne R, Zhang XD and Hersey P: Involvement of endoplasmic reticulum stress in Docetaxel-induced JNK-dependent apoptosis of human melanoma. Apoptosis 13: 1505-1512, 2008.

27. Chen YJ, Su JH, Tsao CY, Hung CT, Chao HH, Lin JJ, Liao MH, Yang ZY, Huang HH, Tsai FJ, et al: Sinulariolide induced hepatocellular carcinoma apoptosis through activation of mitochondrial-related apoptotic and PERK/eIF2 $\alpha / \mathrm{ATF} 4 / \mathrm{CHOP}$ pathway. Molecules 18: 10146-10161, 2013.

28. Li K, Li L, Yang M, Liu H, Boden G and Yang G: The effects of fibroblast growth factor-21 knockdown and over-expression on its signaling pathway and glucose-lipid metabolism in vitro. Mol Cell Endocrinol 348: 21-26, 2012.

29. Lips DJ, Bueno OF, Wilkins BJ, Purcell NH, Kaiser RA, Lorenz JN, Voisin L, Saba-El-Leil MK, Meloche S, Pouysségur J, et al: MEK1-ERK2 signaling pathway protects myocardium from ischemic injury in vivo. Circulation 109: 1938-1941, 2004.
30. Bourke L, McCormick J, Taylor V, Pericleous C, Blanchet B, Costedoat-Chalumeau N, Stuckey D, Lythgoe MF, Stephanou A and Ioannou Y: Hydroxychloroquine protects against cardiac ischaemia/reperfusion injury in vivo via enhancement of ERK1/2 phosphorylation. PLoS One 10: e0143771, 2015.

31. Yang C, Jin C, Li X, Wang F, McKeehan WL and Luo Y: Differential specificity of endocrine FGF19 and FGF21 to FGFR1 and FGFR4 in complex with KLB. PLoS One 7: e33870, 2012.

32. Wang Z, Wang Y, Ye J, Lu X, Cheng Y, Xiang L, Chen L, Feng W, Shi $\mathrm{H}, \mathrm{Yu} \mathrm{X}$, et al: bFGF attenuates endoplasmic reticulum stress and mitochondrial injury on myocardial ischaemia/reperfusion via activation of PI3K/Akt/ERK1/2 pathway. J Cell Mol Med 19: 595-607, 2015.

33. Hu P, Han Z, Couvillon AD and Exton JH: Critical role of endogenous Akt/IAPs and MEK1/ERK pathways in counteracting endoplasmic reticulum stress-induced cell death. J Biol Chem 279: 49420-49429, 2004.

34. Jiang $X$, Zhang $C$, Xin $Y$, Huang Z, Tan Y, Huang $Y$, Wang $Y$, Feng W, Li X, Li W, et al: Protective effect of FGF21 on type 1 diabetes-induced testicular apoptotic cell death probably via both mitochondrial- and endoplasmic reticulum stress-dependent pathways in the mouse model. Toxicol Lett 219: 65-76, 2013. 